\title{
Sparen: Zuerst bei den Strukturen, zuletzt bei den Patienten
}

\author{
Jürg Schlup \\ Dr. med., Präsident der FMH
}

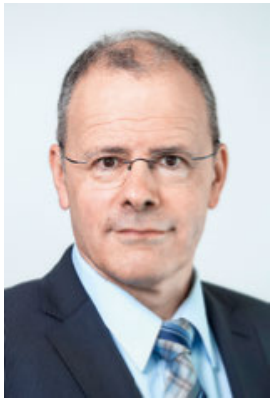

Referenzen

1 Kostendämpfungsmassnahmen zur Entlastung der obligatorischen Krankenpflegeversicherung. Bericht der Expertengruppe. 24. August 2017.

2 Gemeinsame Medienmitteilung der FMH, $\mathrm{H}+$, Interpharma, pharmaSuisse, SPO und santésuisse. Bern, 25. Oktober 2017; Globalbudgets sind leichtfertige Experimente zu Lasten der Patientinnen und Patienten.

3 Adrian Riklin. Aufschrei der Profiteure. Weltwoche 44/2017 vom 2.11.2017

4 Medienmitteilung des BAG vom 2.9. 2016 Mengenwachstum im Gesundheitswesen eindämmen - zusätzliche Massnahmen nötig.

Wille N, Glarner

Schlup J. Die FMH zur

Dämpfung des Kosten-

anstiegs im Gesund-

heitswesen. Schweiz

Ärztezeitung.

2018;99(8):224-6.

6 Meyer, B. Boni-die Position der FMH. Schweiz Ärztezeitung. 2013;94: 51/ 52; S. 1935-7.

Klaus G; Dank einheitlicher Finanzierung mehr Effizienz; Helsana Standpunkt 4/16; S.3-5

OBSAN, Analyse des International Health Policy Survey (IHP) 2015 und OBSAN, Analyse des International Health

Policy Survey (IHP) 2012.
Der im Oktober 2017 vom Eidgenössischen Departement des Inneren (EDI) vorgelegte Expertenbericht «Kostendämpfungsmassnahmen zur Entlastung der obligatorischen Krankenpflegeversicherung» [1] hat bislang vor allem wegen seiner Forderung einer «verbindlichen Zielvorgabe für das OKP-Kostenwachstum» [1] Aufmerksamkeit generiert. Weil solche Globalbudgets die Qualität und die Zugänglichkeit der Gesundheitsversorgung zwangsläufig verschlechtern, positionierten sich Verbände der Patienten, Ärzte, Spitäler, Krankenkassen, Pharmaindustrie und Apotheker gemeinsam klar gegen die Einführung undifferenzierter Kostendeckel [2]. Wer dies als «Aufschrei der Profiteure» [3] verunglimpft, verkennt, dass all diese Akteure täglich einen Beitrag zu einem der international besten Gesundheitswesen leisten, damit in allererster Linie einer profitiert: der Patient.

Der Vorschlag der Experten zukünftig die Gesundheitsversorgung über Budgets zu beschränken, überrascht nicht, denn bereits der Auftrag des EDI hatte vorgegeben, «Steuerungsinstrumente bei den Budgets oder der Menge der zu erbringenden Leistungen" [4] vor allem aus Deutschland und den Niederlanden zu analysieren. Wenn man von dieser Tendenz absieht, grundsätzlich ein "stärkeres Eingreifen" [1] und eine "Gesamtsteuerung" [1] des Staates zu fordern, finden sich unter den insgesamt 38 aufgeführten Massnahmen jedoch auch viele zielführende Ansätze. Dass sich mehrere Forderungen des Expertenberichts sogar mit der Stossrichtung von FMH-Positionen zum Kostendämpfungspotential im Gesundheitswesen decken, zeigt ein Beitrag auf Seite 224 in dieser Ausgabe [5]. Aus ärztlicher Sicht sind in erster Linie Kostendämpfungsmassnahmen zu verfolgen, die Effizienz steigern ohne die Versorgung zu beeinträchtigen. Denn insbesondere die Strukturen und die Finanzierung unseres Gesundheitswesens bergen ungenutztes Potential zur Kostendämpfung. Darum begrüsst die FMH auch, dass der Expertenbericht die Kleinräumigkeit unserer 26 Gesundheitssysteme kritisiert und bspw. eine überregionale Spitalplanung oder die Lösung der Governance-Probleme der Kantone anmahnt.
Auch Spitäler, die mengenbezogene Boni bezahlen, von Spitallisten auszuschliessen, könnte einen von der FMH seit Jahren kritisierten Missstand beheben [6] Strukturell anzusetzen hiesse aber auch mittels einer konsequenten einheitlichen Finanzierung Effizienzpotentiale von jährlich etwa drei Milliarden Franken zu realisieren [7] - ohne dafür Versorgungsleistungen zu reglementieren oder einen neuen, steuernden Verwaltungsapparat schaffen zu müssen. Hier bleibt der Expertenbericht leider hinter den bestehenden Möglichkeiten zurück.

Verbesserte Strukturen und eine einheitliche Finanzierung könnten viel ungenutztes Effizienzpotential ausschöpfen

Ein weiteres im Expertenbericht und auch allgemein von der Politik weitgehend ignoriertes, aber gewichtiges Effizienzpotential liegt in der Reduktion der administrativen Belastung. Alleine um den ständigen Zuwachs an Patientendokumentation bewältigen zu können, braucht es in der Schweiz jedes Jahr rund 100 zusätzliche Spitalärztinnen und -ärzte mit Vollzeitpensum [5]. Und auch im ambulanten Bereich kann nicht einmal mehr die Hälfte der Grundversorger mindestens 75\% ihrer Arbeitszeit mit Patientenkontakten verbringen [8]. Wer Effizienz steigern und Kosten senken möchte, muss die administrative Belastung reduzieren - und keine neue schaffen.

Wer Kosten dämpfen will, muss die administrative Belastung reduzieren - und keine neue schaffen

Die Kostendämpfungsvorschläge der FMH [5] zeigen auf, wo Einsparungen ohne Einschränkungen der Patientenversorgung realisierbar sind. Dies sollte auch bei der Umsetzung von Massnahmen des Expertenberichts im Vordergrund stehen. Denn solange ineffiziente Strukturen und ein unzulängliches Finanzierungssystem fortbestehen, ist es kaum zu rechtfertigen notwendige Versorgungsleistungen zu reduzieren. 